\title{
A closer look at the relationship among accelerometer-based physical activity metrics: ICAD pooled data
}

Soyang Kwon ${ }^{1 *}$, Lars Bo Andersen ${ }^{2}$, Anders Grøntved ${ }^{3}$, Elin Kolle ${ }^{4}$, Greet Cardon ${ }^{5}$, Rachel Davey ${ }^{6}$, Susi Kriemler ${ }^{7}$, Kate Northstone ${ }^{8}$, Angie S. Page ${ }^{9}$, Jardena J. Puder ${ }^{10}$, John J. Reilly ${ }^{11}$, Luis B. Sardinha ${ }^{12}$,

Esther M. F. van Sluijs ${ }^{13}$ and Kathleen F. Janz ${ }^{14}$

\begin{abstract}
Background: Accelerometers are widely used to assess child physical activity (PA) levels. Using the accelerometer data, several PA metrics can be estimated. Knowledge about the relationships between these different metrics can improve our understanding of children's PA behavioral patterns. It also has significant implications for comparing PA metrics across studies and fitting a statistical model to examine their health effects. The aim of this study was to examine the relationships among the metrics derived from accelerometers in children.
\end{abstract}

Methods: Accelerometer data from 24,316 children aged 5 to 18 years were extracted from the International Children's Accelerometer Database (ICAD) 2.0. Correlation coefficients between wear time, sedentary behavior (SB), light-intensity PA (LPA), moderate-intensity PA (MPA), vigorous-intensity PA (VPA), moderate- and vigorous-intensity PA (MVPA), and total activity counts (TAC) were calculated.

Results: TAC was approximately $22 \times 10^{3}$ counts higher $(p<0.01)$ with longer wear time ( 13 to $18 \mathrm{~h} /$ day) as compared to shorter wear time ( 8 to $<13 \mathrm{~h} /$ day), while MVPA was similar across the wear time categories. MVPA was very highly correlated with TAC $(r=.91 ; 99 \% \mathrm{Cl}=.91$ to .91$)$. Wear time-adjusted correlation between SB and LPA was also very high $(r=-.96 ; 99 \% \mathrm{Cl}=-.96,-95)$. VPA was moderately correlated with MPA $(r=.58$; $99 \% \mathrm{Cl}=.57, .59)$.

Conclusions: TAC is mostly explained by MVPA, while it could be more dependent on wear time, compared to MVPA. MVPA appears to be comparable across different wear durations and studies when wear time is $\geq 8 \mathrm{~h} /$ day. Due to the moderate to high correlation between some PA metrics, potential collinearity should be addressed when including multiple PA metrics together in statistical modeling.

Keywords: ICAD, Children, Adolescents, ActiGraph, Total activity counts, Sedentary, Physical activity measurement

\section{Background}

Accelerometers have become a widely used tool to assess physical activity (PA) levels among children. Using accelerometer data, several important public health-related PA metrics, including time spent in sedentary behavior (SB), light-intensity PA (LPA), moderate-intensity PA

\footnotetext{
* Correspondence: skwon@luriechildrens.org

${ }^{1}$ Ann \& Robert H. Lurie Children's Hospital of Chicago Stanley Manne

Children's Research Institute, 225 E Chicago Ave, Box 157, Chicago, IL 60611, USA

Full list of author information is available at the end of the article
}

(MPA), vigorous-intensity PA (VPA), and moderate- to vigorous-intensity PA (MVPA), can be estimated. Daily accumulated accelerometer counts (total activity counts; TAC) has also been suggested as a metric of total PA volume [1-3]. TAC can be conceptualized as a proxy of the total PA volume that encompasses the frequency, intensity, and duration of activity bouts [2]. Despite the conceptual distinctions between TAC and PA intensity metrics, there is a knowledge gap in how TAC is related to time spent in individual PA intensity categories and if wear time affects TAC differently than the individual PA

(C) The Author(s). 2019 Open Access This article is distributed under the terms of the Creative Commons Attribution 4.0 International License (http://creativecommons.org/licenses/by/4.0/), which permits unrestricted use, distribution, and 
intensity categories. For example, it is probable that TAC is mostly explained by accelerometer counts collected during MVPA. Also, TAC could be more dependent on wear time than MVPA.

Accelerometer wear time is a key variable that could significantly impact the accelerometer-derived PA metrics. Although researchers apply an accelerometer data inclusion criterion, for example, at least 8 wear hours per day, to estimate a PA level that can reflect all-day $\mathrm{PA}$, still there are wide variations in wear time from $8 \mathrm{~h}$ to $24 \mathrm{~h}$ per day. Thus, researchers often implement additional approaches (e.g., adjust for wear time in statistical modeling [4]) to standardize/adjust PA metrics within a study population. However, a wear timedependent PA metric has a limitation when comparing the results across studies that have different wear times. That metric may require additional processing to standardize the metric for a comparison. Given that children spend $\geq 90 \%$ of waking time in lower intensity activities such as SB and LPA [5], longer wear time during waking time would capture more SB and LPA. Therefore, it can be hypothesized that TAC, which includes SB and LPA, are more dependent on wear time than MVPA.

As diverse activity metrics can now be calculated using accelerometer data beyond MVPA, which was traditionally the sole focus of PA research when using PA questionnaires, more recent studies have examined the health effects of these diverse accelerometer data-derived metrics. Some, but not all studies [6-8], report that independent of MVPA time, sedentary time is associated with poor health outcomes $[9,10]$, while LPA time is associated with favorable health outcomes $[5,10,11]$. From this perspective, determining which PA behaviors/ metrics are more important has become a great public health concern $[6,8,12,13]$. Although each of the PA intensity metrics conceptually represents a distinct behavior with potentially unique determinants and ascertaining their effects has significant clinical implications, quantifying their independent effects is a challenge. First, because the sum of the accelerometer data-derived metrics (i.e., sum of MVPA, LPA, and SB) results in a finite total accelerometer wear time and time spent in one activity level necessarily displaces time spent in at least another activity level [13], these metrics are inherently co-dependent. Second, because SB, LPA, MPA, and VPA behaviors/metrics are often correlated with each other in empirical data $[14,15]$, this could cause a collinearity problem in a multivariable regression model that includes multiple PA metrics together as predictors. Even when using an alternative approach that fits a regression model for each of the PA intensity metrics individually [12], the results have the limitation of not accounting for the effects of other PA intensity metrics. Knowledge about the relationships between the accelerometer data-derived PA metrics will help to improve our understanding of PA behavior patterns among children and provide more coherent methodological recommendations in the use of PA metrics.

For this study, we had the following hypotheses: (1) TAC is highly correlated with MVPA and mostly explained by MVPA, (2) TAC is more dependent on wear time than MVPA, and (3) some PA intensity metrics are strongly correlated with each other. To test these hypotheses, this study examined the relationships among accelerometer-derived PA metrics in children. These relationships were further examined by individual characteristics, such as sex, age, mother's education level, and obesity status, which have been reported to be associated with PA levels in previous studies [16-18]. The stratified analyses were conducted to ensure the correlations among PA intensity metrics are similar and therefore not confounded by the individual characteristics. This study also examined a correlation between wear time and PA metrics.

\section{Methods \\ Study participants}

This study used data from the International Children's Accelerometer Database (ICAD) 2.0. ICAD, an international pooled database for 20 studies that collected ActiGraph accelerometer data among children. Of the 20 studies, the CHAMPS-US study that conducted three waves of accelerometer assessment within two weeks was excluded from this study. Of the 19 included studies, 12 were conducted in European countries, four in the United States, two in Australia, and one in Brazil. Six were cross-sectional studies, 10 were cohort studies, and four were intervention studies. ICAD obtained and processed raw ActiGraph accelerometry data files from the partner studies. Details on the design and methods of ICAD are described elsewhere [19, 20]. The ICAD 2.0 included 51,434 accelerometer assessments from the 19 studies. For the current analyses, we excluded the post-intervention data of the four intervention studies $(n=9687)$. We further excluded data of participants whose age value was higher than $18(n=2)$, lower than 5 $(n=212)$, or missing $(n=949)$. Because the Magic study only included participants aged 3 and 4 years old, all of the Magic participants were excluded based on the age eligibility criterion. Next, we excluded the spurious ActiGraph data $(n=520)$ as described in Sherar et al. [19] After additionally removing 4435 assessments with $<3$ valid accelerometer wear days (valid wear day was defined as $\geq 480 \mathrm{~min}$ of valid wear time between 6:00 $\mathrm{AM}$ and midnight (12:00 AM) and total activity counts $<1000,000$ counts), 35,629 assessments from 24,316 children aged 5 to 18 years were identified. Of those, 
only the first valid assessment data $(n=24,316)$ were included in the current data analysis.

\section{Data elements}

The ICAD raw accelerometer data were processed using specifically developed and commercially available software (KineSoft, Saskatchewan, Canada). This process has been described in detail elsewhere [12]. Briefly, accelerometry data files were (re)integrated to 60-s epochs. Non-wear time was defined as consecutive zero counts for $\geq 60 \mathrm{~min}$, allowing for two minutes of non-zero interruptions [21]. For the present analysis, we only used accelerometer data collected between 6:00 AM and midnight to (partially) exclude the data collected during nighttime sleep. Accelerometer-derived metrics analyzed included wear time (minutes/day) and TAC (accumulated vertical axis accelerometer counts/day), as well as SB (accumulated time in minutes/day with 0 to 100 accelerometer counts/minute), LPA (accumulated time in minutes/day with 101 to 2295 accelerometer counts/minute), MPA (accumulated time in minutes/day with 2296 and $4011 \mathrm{cpm}$ ), VPA (accumulated time in minutes/day with $\geq 4012 \mathrm{cpm}$ ), and MVPA (accumulated time in minutes/day with $\geq 2296 \mathrm{cpm}$ ), based on Evenson's cut-points [22, 23].

ICAD harmonized mother's education level data into three categories: (1) up to and including completion of compulsory education, (2) some post-compulsory education or vocational training, and (3) completed undergraduate or postgraduate education. The detailed information for the harmonization process can be found at http://www.mrc-epid.cam.ac.uk/research/studies/icad/ data-harmonisation/. Five of the 18 studies did not collect/provide mother's education data. An obesity variable (obese vs. non-obese) was created based on the ageand sex-specific body mass index (BMI) cut-points that correspond to BMI of $30 \mathrm{~kg} / \mathrm{m}^{2}$ for age 18 years [24].

\section{Statistical analysis}

All analyses were conducted in SAS 9.4. (Cary, NC). Before pooled data analyses, the heterogeneity between the studies was examined by comparing the correlation coefficients between accelerometer-derived PA metrics for each study (Additional file 1: Table S1). The results were considered consistent across the studies, and therefore we proceeded to pooled analyses. Among the 18 studies, the median sample size was 1103 . Ten studies had a sample size larger than 1000. Studies with more than 1000 participants were weighted so that the weighted sample size became 1000 . We used this approach, rather than simple inverse variance weighting, to allow for more contribution of the larger studies, and, at the same time, to avoid domination by a very large study (i.e., a study with 6514 participants).
Descriptive analysis was conducted for accelerometerderived metrics. Because the range of PA metrics varied greatly by age, we calculated correlation coefficients of wear time and PA metrics by age group ( 5 to 9,10 to 12 , 13 to 15 , and 16 to 18 years). To test the first hypothesis, we conducted linear regression analysis for TAC predicted by MVPA (Model 1), and then further by wear time (Model 2) or by LPA residuals (Model 3). Standardized LPA residuals were created by regressing MVPA on LPA, to account for the correlation between LPA and MVPA. Correlation analyses between PA metrics were conducted, separately by age group, sex, mother's education, obesity, type of assessment day (weekday and weekend day), and wear time ( 8 to $<13$ and $13-18 \mathrm{~h} /$ day). We further calculated the proportion of VPA within MVPA (VPA minutes $\div$ MVPA minutes $\times 100$ ) for each of the MVPA level categories $(<20,20$ to $<40,40$ to $<60,60$ to $<80$, and $\geq 80 \mathrm{~min} /$ day) to examine whether more active children were proportionally more involved in VPA. A significance level was set at 0.01 , and $99 \%$ confidence intervals $(\mathrm{CI})$ were calculated.

\section{Results}

Among 24,316 participants, $10.7 \%$ had weekday data only, and $7.3 \%$ were categorized as obese. The median number of valid wear days was 6 days (interquartile range of 4 to 7 days).

Means and 99\% CIs of accelerometer-derived metrics are presented in Table 1. Mean SB, LPA, and TAC were significantly higher among children who wore a monitor for 13 to $16 \mathrm{~h}$ per day, compared to those who did for 8 to $<13 \mathrm{~h}$ per day. However, MPA and VPA were similar across the wear time categories. Among boys, while MVPA level was maintained until age 10-12 years and then declined, TAC declined over time, being the highest at age 5-9 years and the lowest at age 16-18 years.

\section{Correlation coefficients between wear time and PA metrics}

We describe the correlation levels based on Mukaka's suggestion: 'negligible' for $r=.0$ to .3; 'low' or "weak" for $r=.3$ to .5 ; 'moderate' for $r=.5$ to .7 ; 'high' for $r=.7$ to .9; and 'very high' for $r=.9$ to 1.0 [25]. As presented in Table 2, wear time was correlated with SB and LPA at a low to moderate level. The correlations of wear time with TAC and MVPA were both at a negligible level, although the correlation coefficients of wear time with TAC were significantly higher than those with MVPA.

\section{Correlation coefficients between PA metrics}

As shown in Table 3, although the correlation between SB and LPA was overall moderate $(r=-.58)$, when it was examined by wear time, it became high $(r=-.77$ and 
Table 1 Means and 99\% confidence intervals of accelerometer-derived metrics

\begin{tabular}{|c|c|c|c|c|c|c|c|}
\hline & $\begin{array}{l}\text { Unweighted } \\
\text { sample }\end{array}$ & $\begin{array}{l}\text { Wear } \\
\text { minutes }\end{array}$ & $\begin{array}{l}\text { Sedentary } \\
\text { minutes }\end{array}$ & LPA minutes & $\begin{array}{l}\text { MPA } \\
\text { minutes }\end{array}$ & $\begin{array}{l}\text { VPA } \\
\text { minutes }\end{array}$ & TAC $\times 1000$ \\
\hline & \multicolumn{3}{|l|}{$n$} & \multicolumn{4}{|l|}{ Mean $(99 \% \mathrm{Cl})$} \\
\hline All & 24,316 & $776(774,777)$ & $382(380,383)$ & $346(345,347)$ & $35(34,35)$ & $13(13,13)$ & $417(415,420)$ \\
\hline \multicolumn{8}{|l|}{ Age and sex* } \\
\hline 5-9yr. boy & 2558 & $758(754,763)$ & $303(298,307)$ & $395(392,399)$ & $45(44,46)$ & $16(15,16)$ & $518(510,525)$ \\
\hline $5-9 y r$. girl & 2740 & $755(751,759)$ & $306(302,310)$ & $400(397,404)$ & $37(36,37)$ & $12(11,12)$ & $467(460,473)$ \\
\hline 10-12yr. boy & 4547 & $768(765,771)$ & $348(345,351)$ & $358(355,360)$ & $45(44,46)$ & $17(17,18)$ & $488(483,493)$ \\
\hline 10-12 yr. girl & 6701 & $769(767,772)$ & $375(373,378)$ & $354(352,356)$ & $30(30,30)$ & $11(10,11)$ & $392(388,395)$ \\
\hline 13-15 yr. boy & 2810 & $804(799,808)$ & $445(440,450)$ & $308(304,312)$ & $33(33,33)$ & $17(17,18)$ & $399(392,406)$ \\
\hline 13-15 yr. girl & 3160 & $799(795,803)$ & $472(468,476)$ & $292(288,295)$ & $26(25,26)$ & $10(10,11)$ & $317(311,322)$ \\
\hline 16-18yr. boy & 872 & $790(781,798)$ & $459(451,468)$ & $288(281,296)$ & $27(26,29)$ & $15(14,16)$ & $353(341,365)$ \\
\hline 16-18yr. girl & 922 & $788(781,796)$ & $481(474,489)$ & $279(273,286)$ & $20(19,21)$ & $7(7,8)$ & $274(265,283)$ \\
\hline \multicolumn{8}{|l|}{ Mother's education level** } \\
\hline$<=$ compulsory education & 6192 & $767(764,769)$ & $356(353,359)$ & $361(359,364)$ & $36(35,36)$ & $14(13,14)$ & $437(432,442)$ \\
\hline $\begin{array}{l}\text { Some post-compulsory } \\
\text { education or vocational }\end{array}$ & 4766 & $777(773,780)$ & $368(365,372)$ & $360(357,362)$ & $35(35,36)$ & $13(13,14)$ & $431(426,436)$ \\
\hline$>=$ completed undergraduate & 4090 & $778(775,781)$ & $375(371,379)$ & $354(351,357)$ & $35(34,36)$ & $14(14,15)$ & $431(425,437)$ \\
\hline \multicolumn{8}{|l|}{ Obesity status*** } \\
\hline Non-obese & 21,728 & $777(775,778)$ & $381(379,382)$ & $347(346,349)$ & $35(35,35)$ & $14(14,14)$ & $422(419,425)$ \\
\hline Obese & 1767 & $767(762,772)$ & $392(385,398)$ & $338(334,343)$ & $28(27,29)$ & $9(8,9)$ & $363(356,371)$ \\
\hline \multicolumn{8}{|l|}{ Type of day } \\
\hline Weekday & 24,316 & $795(793,796)$ & $394(392,396)$ & $349(348,351)$ & $37(37,37)$ & $14(14,15)$ & $433(430,435)$ \\
\hline Weekend & 21,698 & $732(730,734)$ & $352(350,354)$ & $341(339,342)$ & $29(28,29)$ & $11(10,11)$ & $382(379,385)$ \\
\hline \multicolumn{8}{|l|}{ Wear time } \\
\hline $8-<13 \mathrm{~h} /$ day & 12,817 & $714(713,716)$ & $337(335,339)$ & $330(329,332)$ & $34(34,34)$ & $13(12,13)$ & $407(404,410)$ \\
\hline 13-18 h/day & 11,499 & $844(843,846)$ & $431(429,434)$ & $364(362,366)$ & $35(35,36)$ & $14(14,14)$ & $429(425,432)$ \\
\hline
\end{tabular}

${ }^{*}$ n for missing $=6$

**n for missing $=9268$

***n for missing $=821$

$\mathrm{Cl}$, confidence interval; LPA, light-intensity physical activity; MPA, moderate-intensity physical activity; TAC, total activity counts; VPA, vigorous-intensity physical activity

- .78 by wear time categories) or very high (wear time-adjusted $r=-.96)$. The overall correlation coefficient between MVPA and LPA was .32. Regardless of age, sex, mother's education, obesity status, type of day assessed, and length of wear time, MVPA was correlated with TAC at a very high level (overall $r=.91$ ). The correlation coefficient between MPA and VPA was overall .58. In further exploration, we found a trend that the proportion of VPA within MVPA increased as MVPA increased (Fig. 1), indicating that children who are more active engage in proportionally more VPA.

\section{Linear regression models for TAC predicted by MVPA}

As presented in Table 4, MVPA solely explained 83\% of variation in TAC. Adding the wear time variable improved the $\mathrm{R}^{2}$ only $0.4 \%$, although one-minute longer wear time was significantly associated with 100-count higher TAC. Adding the LPA variable improved the $\mathrm{R}^{2}$ by $12 \%\left(R^{2}=0.95\right)$.

\section{Discussion}

This study examined the relationships among accelerometer-derived PA metrics, using data from the largest available child accelerometer database. We found that TAC was mostly (83\%) explained by MVPA. We also found that TAC, compared to MVPA, is more dependent on wear time. SB, LPA, and TAC were higher with longer wear time, although MPA, VPA, and MVPA were similar across different wear durations when daily wear time was $8 \mathrm{~h}$ or higher. SB was negatively correlated with LPA at a high level. Our results also indicate that children who are more active engage in proportionally more VPA. 
Table 2 Correlation coefficients of wear time with physical activity metrics

\begin{tabular}{lllllll}
\hline & SB & LPA & MPA & VPA & MVPA \\
\hline All & $r(99 \% \mathrm{Cl})$ & & & & TAC \\
Age & $.57(.56, .58)$ & $.30(.29, .32)$ & $.06(.05, .07)$ & $.05(.04, .07)$ & $.06(.05, .08)$ & $.12(.10, .13)$ \\
\multicolumn{1}{l}{$5-9$ years } & $.53(.52, .55)$ & $.44(.42, .46)$ & $.12(.09, .14)$ & $.08(.05, .11)$ & $.11(.09, .14)$ & $.20(.17, .22)$ \\
$10-12$ years & $.57(.56, .59)$ & $.49(.47, .50)$ & $.10(.08, .12)$ & $.06(.04, .08)$ & $.09(.07, .11)$ & $.19(.18, .21)$ \\
$13-15$ years & $.57(.56, .59)$ & $.44(.42, .46)$ & $.13(.10, .15)$ & $.02(-.01, .05)$ & $.09(.07, .12)$ & $.20(.17, .22)$ \\
$16-18$ years & $.59(.56, .62)$ & $.42(.38, .46)$ & $.19(.15, .23)$ & $.12(.07, .22)$ & $.18(.13, .22)$ & $.28(.24, .32)$ \\
\hline
\end{tabular}

$\mathrm{Cl}$, confidence interval; LPA, light-intensity physical activity; MPA, moderate-intensity physical activity; MVPA, moderate-and vigorous-intensity physical activity; SB, sedentary behavior; TAC, total activity counts; VPA, vigorous- intensity physical activity

Accelerometer data collected in the field are often unable to provide accelerometer data during the whole waking hours (complete data), due to later put-on in the morning or earlier take-off before sleep, for example. Therefore, it would be ideal to use a PA measure that is least affected by data completeness, so that the measure can be directly comparable across studies. If a PA metric is highly variable depending on the level of data completeness (or wear time) and therefore requires complete data to avoid the bias associated with incomplete data, this would decrease the data usability in epidemiologic studies. This would also potentially eliminate the ability to compare the metrics derived from different wear times across studies. The present study found that SB and LPA were correlated with wear time at a low to moderate level. The present study also found that,

Table 3 Correlation coefficients between accelerometer-derived physical activity metrics

\begin{tabular}{|c|c|c|c|c|c|}
\hline & \multirow[t]{2}{*}{ SB \& LPA } & \multirow[t]{2}{*}{ MVPA \& SB } & MVPA \& LPA & MVPA \& TAC & MPA \& VPA \\
\hline & & & \multicolumn{3}{|l|}{$r(99 \% \mathrm{Cl})$} \\
\hline All & $-.58(-.59,-.57)$ & $-.43(-.44,-.42)$ & $.32(.30, .33)$ & $.91(.91, .91)$ & $.58(.58, .59)$ \\
\hline \multicolumn{6}{|l|}{ Age } \\
\hline $5-9$ years & $-.48(-.50,-.46)$ & $.42(-.44,-.39)$ & $.28(.25, .30)$ & $.92(.92, .92)$ & $.65(.63, .67)$ \\
\hline $10-12$ years & $-.39(-.40,-.37)$ & $-.42(-.43,-.40)$ & $.26(.24, .28)$ & $.92(.92, .93)$ & $.65(.63, .66)$ \\
\hline $13-15$ years & $-.45(-.47,-.43)$ & $-.38(-.40,-.35)$ & $.25(.23, .28)$ & $.92(.92, .92)$ & $.52(.51, .54)$ \\
\hline 16-18 years & $-.45(-.49,-.41)$ & $-.22(-.26,-.18)$ & $.18(.14, .23)$ & $.91(.90, .91)$ & $.58(.54, .61)$ \\
\hline \multicolumn{6}{|l|}{ Sex } \\
\hline Boy & $-.57(-.58,-.55)$ & $-.41(-.43,-.39)$ & $.30(.28, .32)$ & $.91(.91, .92)$ & $.51(.50, .53)$ \\
\hline Girl & $-.61(-.62,-.60)$ & $-.40(-.42,-.30)$ & $.34(.32, .35)$ & $.89(.89,89)$ & $.59(.58, .60)$ \\
\hline \multicolumn{6}{|l|}{ Mother's education level } \\
\hline$<=$ Compulsory education & $-.52(-.54,-.50)$ & $-.46(-.48,-.44)$ & $.32(.30, .34)$ & $.92(.92, .93)$ & $.66(.64, .67)$ \\
\hline Some post-compulsory education or vocational & $-.53(-.55,-.51)$ & $-.43(-.45,-.40)$ & $.35(.32,37)$ & $.92(.92, .92)$ & $.63(.61, .64)$ \\
\hline$>=$ Completed undergraduate & $-.61(-.63,-.59)$ & $-.49(-.51,-.47)$ & $.36(.33, .39)$ & $.93(.92, .93)$ & $.64(.62, .66)$ \\
\hline \multicolumn{6}{|l|}{ Obesity status } \\
\hline Non-obese & $-.58(-.59,-.58)$ & $-.43(-.44,-.42)$ & $.32(.30, .33)$ & $.91(.91, .92)$ & $.58(.57, .59)$ \\
\hline Obese & $-.55(-.58,-.52)$ & $-.42(-.45,-.38)$ & $.30(.26, .35)$ & $.89(.88, .90)$ & $.59(.55, .62)$ \\
\hline \multicolumn{6}{|l|}{ Type of day } \\
\hline Weekday & $-.56(-.57,-.55)$ & $-.40(-.41,-.38)$ & $.30(.29, .31)$ & $.91(.91, .91)$ & $.56(.55, .57)$ \\
\hline Weekend & $-.45(-.46,-.44)$ & $-.39(-.40,-.38)$ & $.32(.31, .33)$ & $.91(.90, .91)$ & $.58(.57, .59)$ \\
\hline \multicolumn{6}{|l|}{ Wear time } \\
\hline $8-<13$ h/day & $-.77(-.78,-.76)$ & $-.52(-.53,-.50)$ & $.32(.30, .33)$ & $.91(.91, .92)$ & $.61(.60, .62)$ \\
\hline 13-18 h/day & $-.78(-.79,-.78)$ & $-.49(-.51,-.48)$ & $.31(.29, .33)$ & $.91(.91, .91)$ & $.56(.55, .57)$ \\
\hline Wear time (continuous variable)-adjusted & $-.96(-.96,-.95)$ & $-56(-.57,-.55)$ & $.31(.30, .31)$ & $.91(91, .91)$ & $.58(.57, .59)$ \\
\hline
\end{tabular}

$\mathrm{Cl}$, confidence interval; LPA, light-intensity physical activity; MPA, moderate-intensity physical activity; MVPA, moderate-and vigorous-intensity physical activity; SB, sedentary behavior; TAC, total activity counts; VPA, vigorous-intensity physical activity 


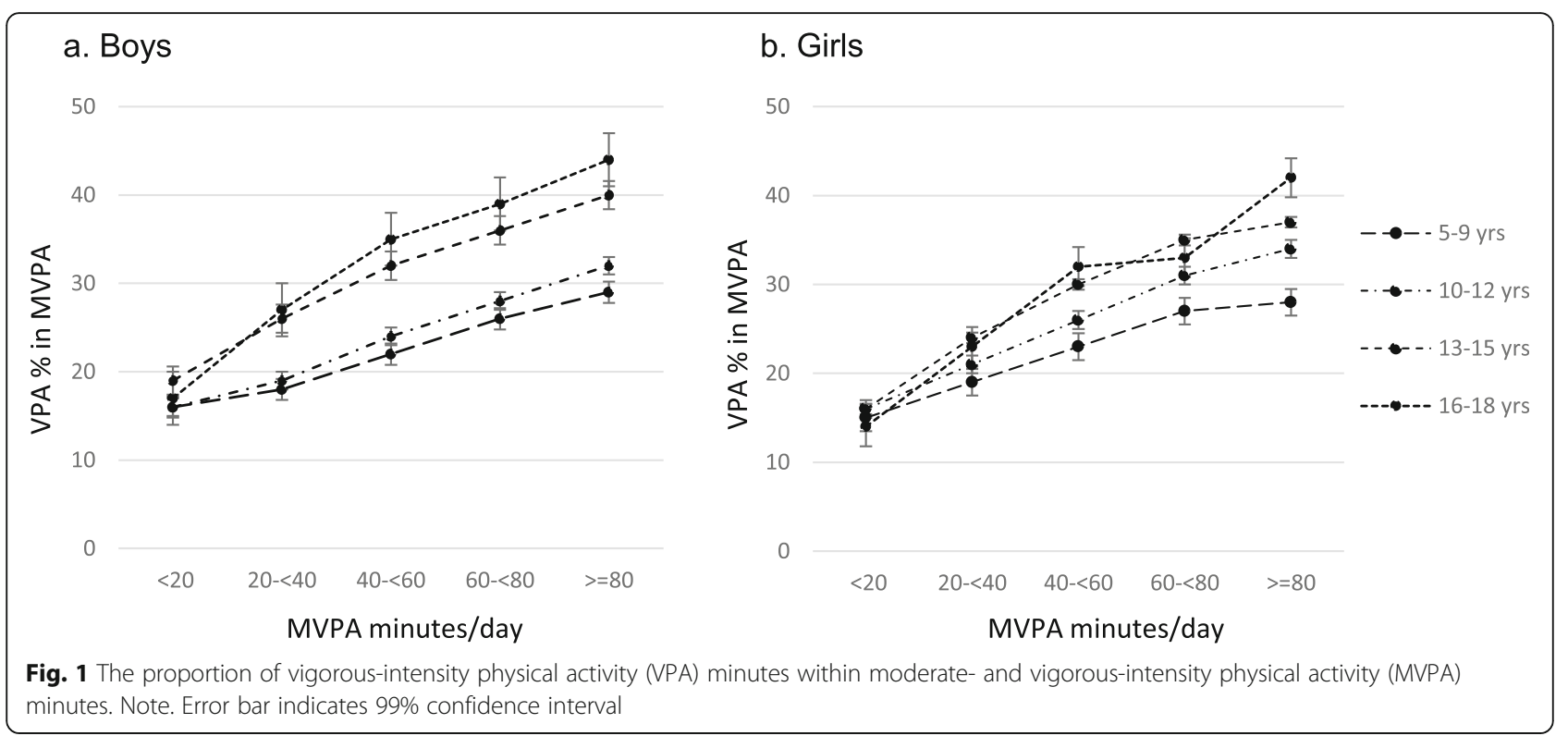

despite the high collinearity between MVPA and TAC and a negligible level of correlation between wear time and TAC, the correlation of wear time with TAC was still higher than the correlation with MPA, VPA, or MVPA, and mean TAC was significantly higher with longer wear time. This implies that TAC could be more affected by data completeness than MPA, VPA, or MVPA. Altogether, these suggest that if most of the data during waking hours are captured (e.g., $\geq 8 \mathrm{~h} /$ day), the bias associated with incomplete data could be minimal for VPA, MPA, and MVPA metrics, but not for SB, LPA, or TAC. This knowledge should increase confidence in study results that examine MPA, VPA, and MVPA, despite inevitable compliance issues. It should also increase confidence that MPA, VPA, and MVPA metrics can be compared across different studies that are likely to have different wear times or data completeness.

Bassett and colleagues [1] have argued that TAC may be a better metric than PA intensity metrics because it incorporates the full continuum of PA intensities. Although this is conceptually true, LPA may not be well reflected in TAC in empirical data. Rather, TAC may be mostly explained by MVPA. In fact, this study revealed that TAC is $83 \%$ explained by MVPA, while it is only $12 \%$ explained by LPA. Our findings emphasize the need for a closer examination of the link between accelerometer counts and PA intensity to better assess whether TAC can be a meaningfully different measure from MVPA in empirical data. For example, despite two decades of accelerometer-measured PA studies, research has not yet closely examined whether an increase in accelerometer counts is proportional to an increase in PA intensity (e.g., expressed in metabolic equivalents). Also, it is an interesting finding that among boys, while MVPA was maintained until preadolescence and then declined, TAC declined over time from age 5 to 18 years. This result could indicate that children maintain MVPA level, but reduce LPA level during preadolescence. However, this result should be further validated to determine whether it is a reflection of true behavioral change during preadolescence or measurement error for MVPA or TAC. Further, as mentioned above, TAC has a

Table 4 Linear regression models to predict daily total activity counts by time spent in moderate- and vigorous-intensity physical activity and wear time

\begin{tabular}{llll}
\hline & Model 1 & Model 2 & Model 3 \\
\hline$R^{2}$ & 0.832 & 0.836 & 0.949 \\
& & Estimate $(99 \% \mathrm{Cl})$ & $164,769(163,602,165,936)$ \\
Intercept & $134,711(162,594,166,828)$ & $87,797(78,716,96,877)$ & $5280(5258,5302)$ \\
MVPA, minutes/day & $5279(5239,5318)$ & $5257(5218,5296)$ & - \\
Wear time, minutes/day & - & $100(89,112)$ & $664(657,672)$ \\
LPA, transformed minutes/day* & - & - & \\
\hline
\end{tabular}

*Standardized residuals of LPA were created by regressing MVPA on LPA. The standardized residual variable was used for regression modeling and the parameter estimate was transformed to express it in LPA minutes/day

$\mathrm{Cl}$, confidence interval; MVPA, moderate-and vigorous-intensity physical activity 
stronger dependency on monitor wear time as compared to MVPA. Altogether, we suggest that the added value of the TAC metric in PA research be further discussed by the research community.

This study reveals an interesting relationship between MVPA and VPA. Children with higher MVPA levels engaged in proportionally more VPA. For example, children who engaged in MVPA for 40 to 60 min daily engaged in VPA for $13 \mathrm{~min}$ (26\% of total MVPA) on average, while children who engaged in MVPA for $\geq 80$ min daily engaged in VPA for $32 \mathrm{~min}$ (33\% of total MVPA), on average. This tendency was much clearer among older children than younger children. For example, among those with $\geq 80 \mathrm{~min}$ of MVPA, the VPA proportion was $29 \%$ for the youngest age group (5 to 9 years), while it was $44 \%$ for the oldest age group (16 to18 years). However, the tendency was not different between boys and girls. Although girls on average had lower MVPA, the proportion of VPA at a given MVPA level was similar between boys and girls. Based on these findings, we presume that sports participation, particularly vigorous-intensity sports participation among older children, largely contributes to children's active lifestyle. However, the interpretation of our results by age groups requires caution since higher VPA proportion among older children could be partly due to use of the same cut-point for VPA across all ages. Our findings also suggest that children who are active are likely to receive the health benefits of VPA, presumably due to their high engagement in VPA.

Examining the effects of the PA metrics is important to establish an evidence base for the health benefits of PA. It is a commonly used statistical approach to include two or more PA intensity metrics in one statistical model to examine their health effects. However, our findings of the substantial correlations between some PA metrics suggest that this approach might violate the non-collinearity assumption required for regression models. To address the collinearity issue, several alternative approaches can be considered. One could divide the study population into subgroups based on PA patterns, for example using cluster analysis, and then compare the health outcomes of those groups. The use of residuals could be another statistical approach. In addition, considering the inherent co-dependency of PA intensity metrics [13], studies that examine the allocation of the 24-h period to the full spectrum of activities, including sleep, SB, LPA, MPA, and VPA, could utilize the compositional data analysis method [5, 26-29].

Several limitations of the study should be acknowledged. First, despite our attempt to (partially) exclude sleep time data by using the data collected only from 6 AM to midnight, this time frame could still include some data during sleep time, which could have biased our results, particularly SB estimation. However, given that only two of the 18 studies (3\% of the sample) used a 24-h accelerometer protocol and the remaining 16 studies used a waking-hour protocol, we expect that the bias would be minimal (Additional file 1: Table S2). Second, although we believe that TAC should be defined as accumulated counts during LPA, MPA, and VPA, but not during $\mathrm{SB}[3,8]$, we used the TAC variable that was defined as accumulated counts during SB through VPA, because the ICAD 2.0 dataset did not contain the TAC variable that only included LPA to VPA. Lastly, with the selection of different cut-points, non-wear criteria, or epoch length, the correlation coefficients that we observed could change. However, we selected to use the most widely accepted accelerometer data reduction methodologies.

\section{Conclusions}

This study provides insights to the pediatric PA research community regarding the selection of accelerometerbased PA metrics and to the investigation of the health effects of PA. This study found that TAC is mostly explained by MVPA, while it could be more dependent on wear time, compared to MVPA. MVPA appears to be comparable across different wear durations and studies when wear time is $\geq 8 \mathrm{~h} /$ day. Because of moderate to high correlations between some PA intensity metrics, potential collinearity should be addressed when including them together in statistical modeling to examine their health effects.

\section{Additional file}

Additional file 1: Table S1. Correlation coefficients between accelerometer-derived physical activity metrics by studies. Table S2. Correlation coefficients between accelerometer-derived metrics for the 16 studies with the walking-hour accelerometer protocol. (DOCX 26 kb)

\section{Abbreviations}

BMI: Body mass index; Cl: Confidence interval; ICAD: International Children's Accelerometer Database; LPA: Light-intensity physical activity; MPA: Moderate-intensity physical activity; MVPA: Moderate- and vigorousintensity physical activity; PA: Physical activity; SB: Sedentary behavior; TAC: Total activity counts; VPA: Vigorous-intensity physical activity

\section{Acknowledgements}

We would like to thank all participants and funders of the original studies that contributed data to ICAD. We gratefully acknowledge the past contributions of Prof Chris Riddoch, Prof Ken Judge, Prof Ashley Cooper and Dr. Pippa Griew to the development of ICAD.

The ICAD was made possible thanks to the sharing of data from the following contributors (study name): Prof LB Andersen, Faculty of Teacher Education and Sport, Western Norway University of Applied Sciences,

Sogndal, Norway (Copenhagen School Child Intervention Study (CoSCIS)); Prof S Anderssen, Norwegian School for Sport Science, Oslo, Norway (European Youth Heart Study (EYHS), Norway); Prof G Cardon, Department of Movement and Sports Sciences, Ghent University, Belgium (Belgium PreSchool Study); Centers for Disease Control and Prevention (CDC), National Center for Health Statistics (NCHS), Hyattsville, MD USA (National Health and Nutrition Examination Survey (NHANES)); Dr. R Davey, Centre for Research 
and Action in Public Health, University of Canberra, Australia (Children's Health and Activity Monitoring for Schools (CHAMPS)); Dr. P Hallal, Postgraduate Program in Epidemiology, Federal University of Pelotas, Brazil (1993 Pelotas Birth Cohort); Prof KF Janz, Department of Health and Human Physiology, Department of Epidemiology, University of lowa, lowa City, US (lowa Bone Development Study); Prof S Kriemler, Epidemiology, Biostatistics and Prevention Institute, University of Zürich, Switzerland (Kinder-Sportstudie (KISS)); Dr. N Møller, University of Southern Denmark, Odense, Denmark (European Youth Heart Study (EYHS), Denmark); Dr. K Northstone, School of Social and Community Medicine, University of Bristol, UK (Avon Longitudinal Study of Parents and Children (ALSPAC)); Dr. A Page, Centre for Exercise, Nutrition and Health Sciences, University of Bristol, UK (Personal and Environmental Associations with Children's Health (PEACH)); Prof R Pate, Department of Exercise Science, University of South Carolina, Columbia, US (Physical Activity in Pre-school Children (CHAMPS-US) and Project Trial of Activity for Adolescent Girls (Project TAAG)); Dr. JJ Puder, Service of Endocrinology, Diabetes and Metabolism, Centre Hospitalier Universitaire Vaudois, University of Lausanne, Switzerland (Ballabeina Study); Prof J Reilly, Physical Activity for Health Group, School of Psychological Sciences and Health, University of Strathclyde, Glasgow, UK (Movement and Activity Glasgow Intervention in Children (MAGIC)); Prof J Salmon, Institute for Physical Activity and Nutrition (IPAN), School of Exercise and Nutrition Sciences, Deakin University, Geelong, Australia (Children Living in Active Neigbourhoods (CLAN) \& Healthy Eating and Play Study (HEAPS)); Prof LB Sardinha, Exercise and Health Laboratory, Faculty of Human Movement, Universidade de Lisboa, Lisbon, Portugal (European Youth Heart Study (EYHS), Portugal); Dr. EMF van Sluijs, MRC Epidemiology Unit \& Centre for Diet and Activity Research, University of Cambridge, UK (Sport, Physical activity and Eating behaviour: Environmental Determinants in Young people (SPEEDY)).

The pooling of the data was funded through a grant from the National Prevention Research Initiative (Grant Number: G0701877) (http://www.mrc.ac. uk/research/initiatives/national-prevention-research-initiative-npri/). The funding partners relevant to this award are: British Heart Foundation; Cancer Research UK; Department of Health; Diabetes UK; Economic and Social Research Council; Medical Research Council; Research and Development Office for the Northern Ireland Health and Social Services; Chief Scientist Office; Scottish Executive Health Department; The Stroke Association; Welsh Assembly Government and World Cancer Research Fund. This work was additionally supported by the Medical Research Council [MC_UU_12015/3; MC_UU_12015/7], The Research Council of Norway (249932/F20), Bristol University, Loughborough University and Norwegian School of Sport Sciences.

\section{Funding}

There are no funding sources to declare.

\section{Availability of data and materials}

None.

\section{Author's contributions}

SK conceived of the study, conducted data analysis, interpreted analysis results, and drafted the manuscript. $\mathrm{KJ}$ helped to draft the manuscript and interpret analysis results. All authors read and approved the final manuscript.

\section{Ethics approval and consent to participate}

All studies included received consent to participate from the legal parent or guardian of participating children.

\section{Consent for publication}

None.

\section{Competing interests}

The authors declare that they have no competing interests.

\section{Publisher's Note}

Springer Nature remains neutral with regard to jurisdictional claims in published maps and institutional affiliations.

\section{Author details}

1Ann \& Robert H. Lurie Children's Hospital of Chicago Stanley Manne Children's Research Institute, 225 E Chicago Ave, Box 157, Chicago, IL 60611, USA. ${ }^{2}$ Faculty of Education, Arts and Sport, Western Norway University of Applied Sciences, Sogndal, Norway. ${ }^{3}$ Department of Sports Science and Clinical Biomechanics, University of Southern Denmark, Odense, Denmark. ${ }^{4}$ Norway, Norwegian School of Sport Science, Oslo, Norway. ${ }^{5}$ Department of Movement and Sports Sciences, Ghent University, 9000 Ghent, Belgium. ${ }^{6}$ Centre for Research \& Action in Public Health Health Research Institute, University of Canberra, Canberra, Australia. ${ }^{7}$ Epidemiology, Biostatistics and Prevention Institute, University of Zürich, Zürich, Switzerland. ${ }^{8}$ Bristol Medical School, University of Bristol, Bristol, UK. ${ }^{9}$ Centre for Exercise, Nutrition and Health Sciences, University of Bristol, Bristol, UK. ${ }^{10}$ Obstetric service, Lausanne University Hospital, Lausanne, Switzerland. ${ }^{11}$ Physical Activity for Health Group, School of Psychological Sciences and Health, University of Strathclyde, Glasgow, UK. ${ }^{12}$ Exercise and Health Laboratory, CIPER, Faculdade de Motricidade Humana, Universidade de Lisboa, Cruz-Quebrada, Portugal. ${ }^{13}$ Centre for Diet and Activity Research (CEDAR) \& MRC Epidemiology Unit, University of Cambridge, Cambridge, UK. ${ }^{14}$ Department of Health and Human Physiology, University of lowa, lowa City, IA, USA.

Received: 27 November 2018 Accepted: 17 April 2019

Published online: 29 April 2019

\section{References}

1. Bassett DR, Troiano RP, McClain JJ, Wolff DL. Accelerometer-based physical activity: total volume per day and standardized measures. Med Sci Sports Exerc. 2015;47(4):833-8.

2. Wolff-Hughes DL, Bassett DR, Fitzhugh EC. Population-referenced percentiles for waist-worn accelerometer-derived total activity counts in U.S youth: 2003 - 2006 NHANES. PLoS One. 2014;9(12):e115915.

3. Maher C, Olds T, Mire E, Katzmarzyk PT. Reconsidering the sedentary behaviour paradigm. PLoS One. 2014;9(1):e86403.

4. Evenson KR, Arredondo EM, Carnethon MR, et al. Physical activity and sedentary behavior among US Hispanic/Latino youth: the SOL youth study. Med Sci Sports Exerc. 2018; Epub ahead of print.

5. Carson V, Tremblay MS, Chaput JP, Chastin SF. Associations between sleep duration, sedentary time, physical activity, and health indicators among Canadian children and youth using compositional analyses. Appl Physiol Nutr Metab. 2016;41(6 Suppl 3):S294-302.

6. Kwon S, Burns TL, Levy SM, Janz KF. Which contributes more to childhood adiposity-high levels of sedentarism or low levels of moderate-throughvigorous physical activity? The lowa bone development study. J Pediatr. 2013;162(6):1169-74.

7. Ekelund U, Luan J, Sherar LB, et al. Moderate to vigorous physical activity and sedentary time and cardiometabolic risk factors in children and adolescents. JAMA. 2012;307(7):704-12.

8. Janz KF, Boros P, Letuchy EM, Kwon S, Burns TL, Levy SM. Physical activity, not sedentary time, predicts dual-energy $X$-ray absorptiometry-measured adiposity age 5 to 19 years. Med Sci Sports Exerc. 2017:49(10):2071-7.

9. Cliff DP, Jones RA, Burrows $T L$, et al. Volumes and bouts of sedentary behavior and physical activity: associations with cardiometabolic health in obese children. Obesity (Silver Spring). 2014;22(5):E112-8.

10. Healy GN, Winkler EA, Brakenridge CL, Reeves MM, Eakin EG. Accelerometerderived sedentary and physical activity time in overweight/obese adults with type 2 diabetes: cross-sectional associations with cardiometabolic biomarkers. PLoS One. 2015;10(3):e0119140.

11. Kwon S, Janz KF, Burns TL, Levy SM. Association between light-intensity physical activity and adiposity in childhood. Pediatr Exerc Sci. 2011;23(2): 218-29.

12. Kuzik N, Carson V, Andersen LB, et al. Physical activity and sedentary time associations with metabolic health across weight statuses in children and adolescents. Obesity (Silver Spring). 2017;25(10):1762-9.

13. Chastin SF, Palarea-Albaladejo J, Dontje ML, Skelton DA. Combined effects of time spent in physical activity, sedentary behaviors and sleep on obesity and cardio-metabolic health markers: a novel compositional data analysis approach. PLoS One. 2015;10(10):e0139984.

14. Healy GN, Matthews CE, Dunstan DW, Winkler EA, Owen N. Sedentary time and cardio-metabolic biomarkers in US adults: NHANES 2003-06. Eur Heart J. 2011;32(5):590-7. 
15. Gomes TN, Hedeker D, Dos Santos FK, et al. Relationship between sedentariness and moderate-to-vigorous physical activity in youth: a multivariate multilevel study. Int J Environ Res Public Health. 2017;14(2):148.

16. Bauman AE, Reis RS, Sallis JF, et al. Correlates of physical activity: why are some people physically active and others not? Lancet. 2012;380(9838):258-71.

17. Hubbard K, Economos CD, Bakun P, et al. Disparities in moderate-tovigorous physical activity among girls and overweight and obese schoolchildren during school- and out-of-school time. Int J Behav Nutr Phys Act. 2016;13:39.

18. Taylor RW, Williams SM, Farmer VL, Taylor BJ. Changes in physical activity over time in young children: a longitudinal study using accelerometers. PLoS One. 2013;8(11):e81567.

19. Sherar $L B$, Griew $P$, Esliger DW, et al. International children's accelerometry database (ICAD): design and methods. BMC Public Health. 2011;11:485.

20. Atkin AJ, Biddle SJH, Broyles ST, et al. Harmonising data on the correlates of physical activity and sedentary behaviour in young people: methods and lessons learnt from the international Children's Accelerometry database (ICAD). Int J Behav Nutr Phys Act. 2017;14(1):174.

21. Troiano RP, Berrigan D, Dodd KW, Masse LC, Tilert T, McDowell M. Physical activity in the United States measured by accelerometer. Med Sci Sports Exerc. 2008:40(1):181-8.

22. Evenson KR, Catellier DJ, Gill K, Ondrak KS, McMurray RG. Calibration of two objective measures of physical activity for children. J Sports Sci. 2008;26(14): 1557-65.

23. Trost SG, Loprinzi PD, Moore R, Pfeiffer KA. Comparison of accelerometer cut-points for predicting activity intensity in youth. Med Sci Sports Exerc. 2011:43(7):1360-8.

24. Cole TJ, Lobstein T. Extended international (IOTF) body mass index cut-offs for thinness, overweight and obesity. Pediatr Obes. 2012;7(4):284-94.

25. Mukaka MM. Statistics corner: a guide to appropriate use of correlation coefficient in medical research. Malawi Med J. 2012;24(3):69-71.

26. Dumuid D, Stanford TE, Martin-Fernández JA, et al. Compositional data analysis for physical activity, sedentary time and sleep research. Stat Methods Med Res. 2018;27(12):3726-38.

27. Dumuid D, Pedišić Ž, Stanford TE, et al. The compositional isotempora substitution model: A method for estimating changes in a health outcome for reallocation of time between sleep, physical activity and sedentary behaviour. Stat Methods Med Res. 2017; Epub ahead of print.

28. Fairclough SJ, Dumuid D, Taylor S, et al. Fitness, fatness and the reallocation of time between children's daily movement behaviours: an analysis of compositional data. Int J Behav Nutr Phys Act. 2017;14(1):64.

29. Grgic J, Dumuid D, Bengoechea EG, et al. Health outcomes associated with reallocations of time between sleep, sedentary behaviour, and physical activity: a systematic scoping review of isotemporal substitution studies. Int J Behav Nutr Phys Act. 2018;15(1):69.

Ready to submit your research? Choose BMC and benefit from:

- fast, convenient online submission

- thorough peer review by experienced researchers in your field

- rapid publication on acceptance

- support for research data, including large and complex data types

- gold Open Access which fosters wider collaboration and increased citations

- maximum visibility for your research: over $100 \mathrm{M}$ website views per year

At $\mathrm{BMC}$, research is always in progress.

Learn more biomedcentral.com/submissions 